\title{
Ground state energy of a non-integer number of particles with $\delta$ attractive interactions
}

\author{
Éric Brunet* and Bernard Derrida ${ }^{\dagger}$ \\ Laboratoire de Physique Statistique, École Normale Supérieure, \\ 24, rue Lhomond, 75231 Paris Cédex 05, France
}

Physica A 279 (2000) 395-407

October 29, 2018

\begin{abstract}
We show how to define and calculate the ground state energy of a system of quantum particles with $\delta$ attractive interactions when the number of particles $n$ is non-integer. The question is relevant to obtain the probability distribution of the free energy of a directed polymer in a random medium. When one expands the ground state energy in powers of the interaction, all the coefficients of the perturbation series are polynomials in $n$, allowing to define the perturbation theory for non-integer $n$. We develop a procedure to calculate all the cumulants of the free energy of the directed polymer and we give explicit, although complicated, expressions of the first three cumulants.
\end{abstract}

PACS numbers: 05.40.+j, 02.50.-r, 82.20.-w.

It is our great pleasure to dedicate this work to our friend Joel L. Lebowitz on the occasion of his 70th birthday.

\section{Introduction}

We consider a system of $n$ identical quantum particles on a ring of size $L$ with $\delta$ attractive interactions. If we call $x_{\alpha}$ (for $1 \leq \alpha \leq n$ ) the positions of the particles, the Hamiltonian of this system is

$$
\mathcal{H}=-\frac{1}{2} \sum_{\alpha} \frac{\partial^{2}}{\partial x_{\alpha}^{2}}-\gamma \sum_{\alpha<\beta} \delta\left(x_{\alpha}-x_{\beta}\right),
$$

\footnotetext{
*Eric.Brunet@physique.ens.fr

$\dagger^{\dagger}$ Bernard.Derrida@physique.ens.fr
} 
where $\gamma$ is the strength of the attractive $(\gamma \geq 0)$ interactions. The main goal of the present work is to define and to calculate the ground state energy $E_{0}(n, L, \gamma)$ of (1) when $n$ is not an integer (especially when $n$ is small).

This system of particles in one dimension with $\delta$ interactions has a long history in the theory of exactly soluble models [1, 2, 3, 4, 5. It was first introduced to describe a Bose gas by Lieb and Liniger who calculated by Bethe ansatz the ground state energy and the excitations for repulsive interactions (that is for negative $\gamma$ ) in the thermodynamic limit ( $n$ and $L$ go to infinity keeping $n / L$ constant) [1].

The problem arose also in the theory of disordered systems: the calculation of the free energy of a directed polymer in a random medium in $1+1$ dimensions by the replica method reduces $6,6,8,8,9,10,11$ to finding the ground state energy of (11): if $Z(x, t)$ is the partition function of a directed polymer joining the points $(0,0)$ and $(x, t)$ on a cylinder with periodic boundary conditions $(x+L \equiv x)$

$$
Z(x, t)=\int_{(0,0)}^{(x, t)} \mathcal{D} y(s) \exp \left(-\int_{0}^{t} d s\left[\frac{1}{2}\left(\frac{d y(s)}{d s}\right)^{2}+\eta(y(s), s)\right]\right),
$$

where the random medium is characterised by a Gaussian white noise $\eta(y, t)$

$$
\left\langle\eta(y, t) \eta\left(y^{\prime}, t^{\prime}\right)\right\rangle=\gamma \delta\left(y-y^{\prime}\right) \delta\left(t-t^{\prime}\right)
$$

then the integer moments of $Z(x, t)$ are given for large $t$ by 12

$$
\lim _{t \rightarrow \infty} \frac{1}{t} \ln \left[\frac{\left\langle Z^{n}(x, t)\right\rangle}{\langle Z(x, t)\rangle^{n}}\right]=-E_{0}(n, L, \gamma),
$$

where \langle\rangle denotes the average over the random medium and $E_{0}(n, L, \gamma)$ is the ground state energy of (1). The knowledge of $E_{0}(n, L, \gamma)$ determines for large $t$ the whole distribution of $\ln Z(x, t)$. For example, the variance of $\ln Z(x, t)$ is

$$
\lim _{t \rightarrow \infty} \frac{\left\langle\ln ^{2} Z(x, t)\right\rangle-\langle\ln Z(x, t)\rangle^{2}}{t}=-\left.\frac{\partial^{2} E_{0}(n, L, \gamma)}{\partial n^{2}}\right|_{n=0} .
$$

Of course, to obtain this variance or other characteristics of the distribution of $\ln Z(x, t)$, one should be able to define and to calculate the ground state energy of (11) not only for integer $n$, but for any value of $n$ 13, 14, 15, 16, 17. Moreover, because of (3), the interactions in (11) must be attractive $(\gamma \geq 0)$; So in contrast to the Bose gas initially studied[1], the interactions are attractive and the interesting limit is no longer the thermodynamic limit $n \rightarrow \infty$ but rather the limit $n \rightarrow 0$.

For integer $n$ and $L=\infty$, the $n$ particles form a bound state at energy 4 , [6]

$$
E_{0}(n, \infty, \gamma)=-\gamma^{2} \frac{n\left(n^{2}-1\right)}{24}
$$

Using this formula for non-integer $n$ helped to understand several properties 12 of the distribution of $\ln Z(t)$ when $L$ is infinite. There are however a number 
of difficulties with (6) for non-integer $n$, in particular a problem of convexity: $d^{2} \ln \left\langle Z^{n}\right\rangle / d n^{2}$ should be positive for all $n$, and so (4, 6) cannot be valid at least for negative $n$. We believe that these difficulties are due to the exchange of limit $t \rightarrow \infty$ and $L \rightarrow \infty$ and this is why we try in the present work to determine $E_{0}(n, L, \gamma)$ for finite $L$.

The paper is organised as follows: in section 2, we recall the Bethe ansatz equations which give the ground state energy of (11) for an (integer) number $n$ of particles and we write the integral equation (13) which is a way of solving the coupled non-linear equations of the Bethe ansatz. The main advantage of this integral equation is that both the strength $\gamma$ of the interactions and the number $n$ of particles appear as continuous parameters. In section 3 we solve (13) perturbatively in $c$ (where $c=\gamma L / 2$ ) for arbitrary $n$. We notice that the coefficients in the small $c$ expansion of $E_{0}(n, L, \gamma)$ are all polynomials in $n$, thus allowing to define the expansion even for non-integer $n$. In section 1 , we show how to generate a small $n$ expansion of the solution of (13). We give explicit expressions up to order $n^{3}$ of $E_{0}(n, L, \gamma)$ and we notice that the coefficients of the small $n$ expansion have in general a zero radius of convergence in $c$.

\section{The Bethe ansatz equations}

The Bethe ansatz 18 consists in looking in the region $0 \leq x_{1} \leq \cdots \leq x_{n} \leq L$ for a ground state wave function $\Psi\left(x_{1}, \ldots, x_{n}\right)$ of the form

$$
\Psi\left(x_{1}, \ldots, x_{n}\right)=\sum_{P} A_{P} e^{\frac{2}{L}\left(q_{1} x_{P(1)}+\cdots+q_{n} x_{P(n)}\right)},
$$

where the sum in (7) runs over all the permutations $P$ of $\{1, \ldots, n\}$. The value of $\Psi$ in other regions can be deduced from (7) by symmetries. One can show $3,4,5]$ that (7) is an eigenstate of (1) at energy

$$
E(n, L, \gamma)=-\frac{2}{L^{2}} \sum_{1 \leq \alpha \leq n} q_{\alpha}^{2},
$$

if the $\left\{q_{\alpha}\right\}$ are solutions of the $n$ coupled equations

$$
e^{2 q_{\alpha}}=\prod_{\beta \neq \alpha} \frac{q_{\alpha}-q_{\beta}+c}{q_{\alpha}-q_{\beta}-c}
$$

where

$$
c=\frac{\gamma L}{2} .
$$

(A derivation of (9) can be found in [3]. Note that $i k_{j}$ and $c$ in [3] become here respectively $\frac{2}{L} q_{j}$ and $-\gamma$; the $c$ in [3] and our $c$ defined in (10) are thus different.) Moreover, for $\gamma \neq 0$, all the $q_{\alpha}$ are distinct.

There are a priori many solutions of (9). We look for the ground state, that is the solution $\left\{q_{\alpha}\right\}$ for which (8) is minimal. When $c=0$, the problem 
reduces to $n$ non-interacting particles $\left\{q_{\alpha}\right\}=\{0\}$ (we have periodic boundary conditions). Because the ground state solution is not degenerate, the solution $\left\{q_{\alpha}\right\}$ of (9) must have the symmetry $\left\{q_{\alpha}\right\}=\left\{-q_{\alpha}\right\}$, depend continuously on $c$, and vanish as $c \rightarrow 0$.

Let us introduce the following function of $\left\{q_{\alpha}\right\}$ :

$$
B(u)=\frac{1}{n} e^{\frac{c}{4}\left(u^{2}-1\right)} \sum_{q_{\alpha}} \rho\left(q_{\alpha}\right) e^{q_{\alpha}(u-1)},
$$

where the parameters $\rho\left(q_{\alpha}\right)$ are defined by

$$
\rho\left(q_{\alpha}\right)=\prod_{q_{\beta} \neq q_{\alpha}} \frac{q_{\alpha}-q_{\beta}+c}{q_{\alpha}-q_{\beta}} .
$$

The function $B(u)$ is a rather complicated (but easier to manipulate than the $q_{\alpha}$ ) symmetric function of the ground state solution $\left\{q_{\alpha}\right\}$ of (9). As shown in the appendix, it satisfies the integral equation

$$
B(1+u)-B(1-u)=n c \int_{0}^{u} e^{-\frac{c}{2}\left(v^{2}-u v\right)} B(1-v) B(1+u-v) d v
$$

and the following two conditions

$$
\begin{aligned}
& B(1)=1, \\
& B(u)=B(-u) .
\end{aligned}
$$

Moreover, the energy (8) can be deduced from of $B(u)$ by

$$
E_{0}(n, L, \gamma)=\frac{2}{L^{2}}\left[\frac{n^{3} c^{2}}{6}+\frac{n c^{2}}{12}+\frac{n c}{2}-n B^{\prime \prime}(1)\right] .
$$

The derivation of $(13,14,15,16)$ is given in the appendix. How these relations lead to small $c$ or small $n$ expansions is explained in sections 3 and 6 .

\section{Expansion in powers of $c$}

One could try to solve the equations (9) perturbatively in $c$ but the approach turns out to be quickly complicated 19]. Instead we are going to see that the integral equation (13) is very convenient to obtain $E_{0}(n, L, \gamma)$ for small $c$.

It is known (and easy to check from (91)) that the $q_{\alpha}$ scale like $\sqrt{c}$ for small $c$. Therefore each coefficient $B_{i}(u)$ of the small $c$ expansion of $B(u)$ defined by (11) is a polynomial in $u$.

$$
B(u)=B_{0}(u)+c B_{1}(u)+c^{2} B_{2}(u)+\ldots
$$

Moreover, conditions (14) and (15) impose that all the $B_{i}(u)$ are even, that $B_{0}(1)=1$ and $B_{i}(1)=0$ for any $i \geq 1$. 
At zero-th order in $c$, we find, using (13):

$$
B_{0}(1+u)-B_{0}(1-u)=0
$$

Thus $B_{0}(u)$ and $B_{0}(1+u)$ are both even functions of $u$. As $B_{0}(u)$ is a polynomial and $B_{0}(1)=1$, the only solution is

$$
B_{0}(u)=1 .
$$

We put this back into (13) and get at first order in $c$

$$
B_{1}(1+u)-B_{1}(1-u)=n u .
$$

Again, using the fact that $B_{1}(u)$ is an even polynomial such that $B_{1}(1)=0$, the only possible solution is:

$$
B_{1}(u)=\frac{n}{4}\left(u^{2}-1\right) .
$$

It is easy to see from (13) that at any order in $c$, we have to solve

$$
B_{i}(1+u)-B_{i}(1-u)=\text { "some polynomial odd in } u \text { ", }
$$

and that there is a unique even polynomial solution satisfying $B_{i}(1)=0$. One can generate as many $B_{i}(u)$ as needed to obtain $B(u)$ up to any desired order in $c$.

$$
B(u)=1+\frac{c n\left(-1+u^{2}\right)}{4}+\frac{c^{2} n(1+2 n)\left(-1+u^{2}\right)^{2}}{96}+O\left(c^{3}\right) .
$$

Relation (16) then gives the energy. Up to the fourth order in $c$, we find

$$
\frac{L^{2}}{2} E_{0}(n, L, \gamma)=-n(n-1)\left(\frac{c}{2}+\frac{c^{2}}{12}+\frac{n}{180} c^{3}+\left(\frac{n^{2}}{1512}-\frac{n}{1260}\right) c^{4}+\ldots\right)
$$

\section{Solution for small $n$}

It is clear from section 3 that if we stop the small $c$ expansion of $B(u)$ at a given order, $B(u)$ and $E_{0}(n, L, \gamma)$ are polynomials in $n$. This allows to define the small $c$ expansion of $B(u)$ or of $E_{0}(n, L, \gamma)$ for an arbitrary value of $n$.

Moreover, we can write a small $n$ expansion of $B(u)$ by collecting all the terms proportional to $n^{k}$ in the small $c$ expansion of $B(u)$ and calling the series obtained $b_{k}(u)$. Then,

$$
B(u)=1+n b_{1}(u)+n^{2} b_{2}(u)+\ldots
$$

Conditions (14, 15) impose that $b_{k}(u)=b_{k}(-u)$ and $b_{k}(1)=0$ for all $k \geq 1$. 
We are now going to describe a procedure which leads to a recursion on the $b_{k}(u)$ and allows to write them not only as power series in $c$ but as explicit functions of $c$ and $u$. If we insert (25) into (13), we get at first order in $n$

$$
b_{1}(1+u)-b_{1}(1-u)=c \int_{0}^{u} e^{-\frac{c}{2}\left(v^{2}-u v\right)} d v .
$$

It can be checked that a solution of (26) compatible with the conditions $b_{1}(u)=$ $b_{1}(-u)$ and $b_{1}(1)=0$ is

$$
b_{1}(u)=\sqrt{c} \int_{0}^{+\infty} d \lambda \frac{\cosh \frac{\lambda u \sqrt{c}}{2}-\cosh \frac{\lambda \sqrt{c}}{2}}{\sinh \frac{\lambda \sqrt{c}}{2}} e^{-\frac{\lambda^{2}}{2}} .
$$

There are however other solutions to the difference equation (26): one could add to (27) an arbitrary function $F(u, c)$ even and periodic in $u$ of period 2 and vanishing at $u=1$. If we require that each term in the small $c$ expansion of $b_{1}(u)$ is polynomial in $u$ (as justified in section 3), we see that all the terms of the small $c$ expansion of $F(u, c)$ must be identically zero. For example $F(u, c)=$ $\exp \left(-c^{-1 / 4}\right)(\cos (\pi u)+1)$ is an acceptable function. This already shows that $b_{1}(u)$ given by (27) has indeed for small $c$ expansion the series obtained by collecting all the terms proportional to $n$ in the small $c$ expansion of section 3 .

If the solution $b_{1}(u)$ (27) had a non-zero radius of convergence in $c$, it would be natural to choose the only $b_{1}(u)$ which is analytic in $c$ at $c=0$ by taking $F(u, c)=0$. Unfortunately, this is not the case: by making the change of variable $\lambda^{2}=\mu$, expression (27) appears as the Borel sum of a divergent series 20 .

We found no conclusive reasons why (27) is the solution of (26) we should select. However, one can notice that, when $n$ is an integer, $B(u)$ is analytic in $u$ and goes to zero when $u \rightarrow \pm i \infty$ (see (11)). Here, the $b_{1}(u)$ given by (27) grows like $\ln |u|$ when $u \rightarrow \pm i \infty$. Adding a non-zero periodic $F(u, c)$ would either lead to an exponential growth in the imaginary direction or introduce singularities in the complex $u$ plane. So (27) is the solution of (26), analytic in the whole $u$ plane, which has the slowest growth in the imaginary direction.

The same difficulty of selecting the right solution appears at every order in the expansion in powers of $n$. We are now going to explain the procedure we have used to select one solution. If we insert (25) into (13), we have to solve at any order $k$ in the small $n$ expansion

$$
b_{k}(1+u)-b_{k}(1-u)=\phi_{k}(u),
$$

where $\phi_{k}(u)$ is a function odd in $u$ which can be calculated from the previous orders

$$
\phi_{k}(u)=c \sum_{i=0}^{k-1} \int_{0}^{u} e^{-\frac{c}{2}\left(v^{2}-u v\right)} b_{i}(1-v) b_{k-i-1}(1+u-v) d v .
$$

(For consistency, we use $b_{0}(u)=1$.) It can be checked that a solution to (28) is

$$
b_{k}(u)=\int_{0}^{+\infty} d \lambda \frac{\cosh \frac{\lambda u \sqrt{c}}{2}-\cosh \frac{\lambda \sqrt{c}}{2}}{\sinh \frac{\lambda \sqrt{c}}{2}} a_{k}(\lambda),
$$


where $a_{k}(\lambda)$ is given by

$$
a_{k}(\lambda)=\frac{1}{2 i \pi} \int_{0}^{+\infty} d u \sin \frac{\lambda u}{2} \phi_{k}\left(\frac{i u}{\sqrt{c}}\right) .
$$

Indeed, the verification is a simple matter of algebra. (We convinced ourselves that $b_{k}(u) \sim \ln ^{k}|u|$ and $\phi_{k}(u) \sim \ln ^{k-1}|u| / u$ as $u \rightarrow \pm i \infty$, and that $a_{k}(\lambda) \sim$ $\ln ^{k-1}|\lambda|$ for $\lambda \rightarrow 0$ and $a_{k}(\lambda) \sim \exp \left(-\lambda^{2}(k+1) / 4 k\right)$ for $\lambda \rightarrow \infty$, so that all the integrals in (30, 31) converge.)

As for $b_{1}(u)$, one could add to the $b_{k}(u)$ given by $(30,31)$ an arbitrary function $F_{k}(u, c)$, even and periodic in $u$ of period 2 to obtain the general solution of (28). However, to be consistent with the small $c$ expansion of section 3, the small $c$ expansion of $F_{k}(u, c)$ should be identically zero. Moreover if we want the solution of (28) to be analytic in the whole $u$ plane and not to grow too fast when $u \rightarrow \pm i \infty$, we must take $F_{k}(u, c)=0$.

Expression (27) for $b_{1}(u)$ is in fact a particular case of the procedure (30, 31); when applied to (26), it gives indeed $a_{1}(\lambda)=\sqrt{c} \exp \left(-\lambda^{2} / 2\right)$.

At second order in the small $n$ expansion, we find for $\lambda>0$

$$
a_{2}(\lambda)=c e^{-\frac{\lambda^{2}}{2}}\left[\int_{0}^{\lambda} d \mu e^{-\frac{\mu^{2}}{2}} \frac{2 \cosh \frac{\lambda \mu}{2}-2}{\tanh \frac{\mu \sqrt{c}}{2}}+\int_{\lambda}^{+\infty} d \mu e^{-\frac{\mu^{2}}{2}} \frac{e^{-\frac{\lambda \mu}{2}}-2}{\tanh \frac{\mu \sqrt{c}}{2}}\right] .
$$

The expressions of $b_{3}(u)$ or $a_{3}(\lambda)$ would be much longer to write and higher orders even more complicated. Recursion (29, 30, 31) allows nevertheless to calculate in principle the whole expansion in powers of $n$.

Using relation (16) and the expressions (27) and (32) of $b_{1}(u)$ and $a_{2}(\lambda)$, we find that the energy $E_{0}(n, L, \gamma)$ is given up to order $n^{3}$ :

$$
\begin{aligned}
& \frac{L^{2}}{2} E_{0}(n, L, \gamma)=n\left(\frac{c}{2}+\frac{c^{2}}{12}\right)-n^{2} \frac{c^{3 / 2}}{4} \int_{0}^{+\infty} d \lambda \frac{\lambda^{2}}{\tanh \frac{\lambda \sqrt{c}}{2}} e^{-\frac{\lambda^{2}}{2}}+n^{3} \frac{c^{2}}{6} \\
& -n^{3} \frac{c^{2}}{4} \int_{0}^{+\infty} d \lambda \frac{\lambda^{2} e^{-\frac{\lambda^{2}}{2}}}{\tanh \frac{\lambda \sqrt{c}}{2}}\left[\int_{0}^{\lambda} d \mu e^{-\frac{\mu^{2}}{2}} \frac{2 \cosh \frac{\lambda \mu}{2}-2}{\tanh \frac{\mu \sqrt{c}}{2}}+\int_{\lambda}^{+\infty} d \mu e^{-\frac{\mu^{2}}{2}} \frac{e^{-\frac{\lambda \mu}{2}}-2}{\tanh \frac{\mu \sqrt{c}}{2}}\right]
\end{aligned}
$$

As explained in the introduction, this small $n$ expansion of $E_{0}(n, L, \gamma)$ gives the cumulants of the free energy in the directed polymer problem. Of course, if we expand (33) in powers of $c$, we recover (24).

\section{Conclusion}

In this work, we have developed a method allowing to calculate perturbatively the ground state energy of (11) for a non-integer number $n$ of particles. We first generated for integer $n$ a perturbation series in powers of the interaction $c$. Each term of this series is polynomial in $n$, allowing to define a small $c$ expansion of the energy for non-integer $n$. This series, at least for small $n$, has in general a zero radius of convergence, in contrast to integer $n$ for which the radius of 
convergence of the perturbation theory is non-zero 21]. (For $n=2$, the closest singularities of $E_{0}(2, L, \gamma)$ in the $c$ plane lie at $c \simeq 3.30 \pm i 4.12$.)

We believe that the fact that each term in the perturbation theory is polynomial in $n$ is generic and would be true for an arbitrary pair interaction and in any dimension. As the link (1) to directed polymers is valid in any dimension, it would be useful and interesting to try to recover our results by doing a direct perturbation theory of the Hamiltonian instead of our Bethe ansatz approach (which is limited to $1+1$ dimensions and to a $\delta$ potential) in order to see whether the calculations could be extended to higher dimensions.

Our calculation of the ground state energy for non-integer $n$ is based on the integral equation (13) and the conditions (14, 15). When we tried to solve the problem for small $n$, at each order we had to select a particular solution of a difference equation. We did not find a conclusive reason to justify the solution we selected, apart from some analyticity properties and growth criterion in the complex plane of the variable $u$. It would certainly be interesting to justify our choice (33) by calculating the second and the third cumulants of $\ln Z$ directly (and not only perturbatively to all orders in $c$ ).

In our small $n$ expansion of section 1 , the terms become quickly very complicated. There is however a regime, which corresponds to the large $c$ limit of recursion (29, 30, 31) where one can handle all orders in the small $n$ expansion 19. This allows one to calculate the whole distribution of $\ln (Z(x, t)) / t$ when $t$ is very large and $(1 / t) \ln (Z(x, t) /\langle Z(x, t)\rangle)$ of order $1 / L$. One can then recover 19 the same large deviation function as found for the asymmetric exclusion process 22 , $23,24,25,261$, as expected since the directed polymer problem in $1+1$ dimensions and the asymmetric exclusion process are both representatives of the KPZ equation 27, 12, 28] in dimension 1. This strengthens the conjecture that the solutions to the difference equations we selected in section 1 give indeed the right non-integer moments of the partition function.

From the point of view of the theory of disordered systems, our results give one of the very few examples for which the distribution of $Z$ can be calculated exactly. In particular they could provide a good test of the replica approach and of other variational methods [7, 9, 10, 11.

A simple and interesting phenomenon visible in the present work (which is generic of all kinds of disordered systems with Gaussian disorder) is that the weak disorder expansion (here small $c$ expansion) of non-integer moments of the partition function has a zero radius of convergence whereas integer moments have a non-zero radius of convergence. This is already visible in the trivial example of a single Ising spin $\sigma= \pm 1$ in a random Gaussian field $h$; the partition function at temperature $T$ is $Z=2 \cosh (h / T)$, and it is easy to check that all non-integer moments of the partition function have a zero radius of convergence in $1 / T$.

Acknowledgements We thank François David, Michel Gaudin, Vincent Pasquier, Leonid Pastur, Herbert Spohn and André Voros for useful discussions. 


\section{A Derivation of (13, 14, 15, 16)}

Let us first establish some useful properties of the numbers $\rho\left(q_{\alpha}\right)$ defined by (12). If the $q_{\alpha}$ are the $n$ roots of the polynomial $P(X)$ defined as

$$
P(X)=\prod_{q_{\alpha}}\left(X-q_{\alpha}\right),
$$

it is easy to see that the $\rho\left(q_{\alpha}\right)$ defined in (12) satisfy

$$
\frac{P(X+c)}{P(X)}=1+c \sum_{q_{\alpha}} \frac{\rho\left(q_{\alpha}\right)}{X-q_{\alpha}} .
$$

(The two sides have the same poles with the same residues and coincide at $X \rightarrow \infty$.) Expanding the right hand side of (A.2) for large $X$, we get

$$
\frac{P(X+c)}{P(X)}=1+c \sum_{q_{\alpha}} \frac{\rho\left(q_{\alpha}\right)}{X}\left(1+\frac{q_{\alpha}}{X}+\frac{q_{\alpha}^{2}}{X^{2}}\right)+O\left(\frac{1}{X^{4}}\right) .
$$

On the other hand, using (8, A.1) and the symmetry $\left\{q_{\alpha}\right\}=\left\{-q_{\alpha}\right\}$ we have

$$
P(X)=X^{n}+\frac{L^{2}}{4} E_{0}(n, L, \gamma) X^{n-2}+O\left(X^{n-4}\right),
$$

so that

$$
\frac{P(X+c)}{P(X)}=1+\frac{n c}{X}+\frac{c^{2}\left(\begin{array}{l}
n \\
2
\end{array}\right)}{X^{2}}+\frac{c^{3}\left(\begin{array}{l}
n \\
3
\end{array}\right)-c E_{0}(n, L, \gamma) L^{2} / 2}{X^{3}}+O\left(\frac{1}{X^{4}}\right) .
$$

Comparing (A.3) and (A.5), we get the relations

$$
\begin{aligned}
\sum_{q_{\alpha}} \rho\left(q_{\alpha}\right) & =n, \\
\sum_{q_{\alpha}} q_{\alpha} \rho\left(q_{\alpha}\right) & =c\left(\begin{array}{l}
n \\
2
\end{array}\right), \\
\sum_{q_{\alpha}} q_{\alpha}^{2} \rho\left(q_{\alpha}\right) & =c^{2}\left(\begin{array}{l}
n \\
3
\end{array}\right)-\frac{E_{0}(n, L, \gamma) L^{2}}{2}
\end{aligned}
$$

Moreover, by letting $X= \pm q_{\beta}-c$ in (A.2) we get for any $q_{\beta}$ root of $P(X)$

$$
\frac{1}{c}=\sum_{q_{\alpha}} \frac{\rho\left(q_{\alpha}\right)}{q_{\alpha}-q_{\beta}+c}=\sum_{q_{\alpha}} \frac{\rho\left(q_{\alpha}\right)}{q_{\alpha}+q_{\beta}+c} .
$$

Lastly using the symmetry $\left\{q_{\alpha}\right\}=\left\{-q_{\alpha}\right\}$ and the definition (12), the Bethe ansatz equations (9) reduce to

$$
e^{q_{\alpha}} \rho\left(-q_{\alpha}\right)-e^{-q_{\alpha}} \rho\left(q_{\alpha}\right)=0 .
$$

From the definition (11) of $B(u)$ and the properties (A.6 A.10), it is straightforward to establish (13) 16): the integral equation (13) is a direct consequence

of (11) and (A.9). Properties (14, 15) follow from (11, A.6) and (11, A.10) respectively. Lastly (16) is a consequence of (11, A.6 A.8). 


\section{References}

[1] E. H. Lieb and W. Liniger, Phys. Rev. 130, 1605 (1963).

[2] C. N. Yang and C. P. Yang, J. Math. Phys. 10, 1115 (1969).

[3] M. Jimbo, T. Miwa, Y. Môri, and M. Sato, Physica 1D, 80 (1980).

[4] H. B. Thacker, Reviews of Modern Physics 53, 253 (1981).

[5] M. Gaudin, La Fonction d'Onde de Bethe (Masson, Paris, 1983).

[6] M. Kardar, Nuclear Physics B 290 [FS20], 582 (1987).

[7] M. Mézard and G. Parisi, J. Phys. I France 1, 809 (1991).

[8] J.-P. Bouchaud and H. Orland, J. Stat. Phys. 61, 877 (1990).

[9] Y. Y. Goldschmidt, Nucl. Phys. B393, 507 (1993).

[10] T. Garel and H. Orland, Phys. Rev. B 55, 226 (1997).

[11] D. B. Saakian and T. M. Nieuwenhuizen, J. Phys. I France 7, 1513 (1997).

[12] T. Halpin-Healy and Y.-C. Zhang, Physics Reports 254, 215 (1995).

[13] J. Krug, Adv. Phys. 46, 139 (1997).

[14] T. Halpin-Healy, Phys. Rev. A 44, R3415 (1991).

[15] J. Krug, P. Meakin, and T. Halpin-Healy, Phys. Rev. A 45, 638 (1992).

[16] J. M. Kim, M. A. Moore, and A. J. Bray, Phys. Rev. A 44, 2345 (1991).

[17] Y.-C. Zhang, J. Stat. Phys. 57, 1123 (1989).

[18] H. A. Bethe, Zeitschrift für Physik 71, 205 (1931).

[19] É. Brunet and B. Derrida, Phys. Rev. E 61, (2000).

[20] B. Candelpergher, M. A. Coppo, and E. Delabaere, l'enseignement mathématique 1 (1997).

[21] T. Kato, Perturbation Theory for Linear Operators (Springer Verlag, Berlin, 1966).

[22] B. Derrida and J. L. Lebowitz, Phys. Rev. Lett. 80, 209 (1998).

[23] B. Derrida and C. Appert, J. Stat. Phys. 94, 1 (1999).

[24] D. Kim, Phys. Rev. E 52, 3512 (1995).

[25] D.-S. Lee and D. Kim, Phys. Rev. E 59, 6476 (1999).

[26] C. Appert, Phys. Rev. E 61, 2092 (2000). 
[27] M. Kardar, G. Parisi, and Y.-C. Zhang, Phys. Rev. Lett. 56, 889 (1986).

[28] A. L. Barabási and H. E. Stanley, Fractal Concepts in Surface Growth (CUP, Cambridge, 1995). 